\title{
Nonspreading Solutions and Patch Formation in An Integro-Difference Model With a Strong Allee Effect and Overcompensation
}

\section{Garrett Otto}

SUNY Cortland

\section{William Fagan}

University of Maryland at College Park

Bingtuan Li ( $\boldsymbol{D}$ bing.li@louisville.edu )

University of Louisville https://orcid.org/0000-0002-1444-6201

\section{Research Article}

Keywords: Integro-difference equation, Allee effect, Overcompensation, Nonspreading solution

Posted Date: October 26th, 2021

DOI: https://doi.org/10.21203/rs.3.rs-953137/v1

License: (c) (i) This work is licensed under a Creative Commons Attribution 4.0 International License.

Read Full License 


\title{
Nonspreading Solutions and Patch Formation in an Integro-Difference Model with a Strong Allee Effect and Overcompensation
}

\author{
Garrett Otto* \\ William F. Fagan ${ }^{\dagger}$ \\ Bingtuan $\mathrm{Li}^{\ddagger}$
}

*Department of Mathematics, SUNY Cortland, Cortland, NY 13045, USA (garrett.otto@ cortland.edu).

†Department of Biology, University of Maryland, College Park, MD 20742, USA (bfagan@umd.edu). This author was supported by the National Science Foundation under Grant DMS- 1853465.

${ }^{\ddagger}$ Department of Mathematics, University of Louisville, Louisville, KY 40292, USA (bing.li@louisville.edu). This author was partially supported by the National Science Foundation under Grant DMS-1951482. 


\begin{abstract}
Previous work involving integro-difference equations of a single species in a homogenous environment has emphasized spreading behaviour in unbounded habitats. We show that under suitable conditions, a simple scalar integro-difference equation incorporating a strong Allee effect and overcompensation can produce solutions where the population persists in an essentially bounded domain without spread despite the homogeneity of the environment. These solutions are robust in that they occupy a region of full measure in the parameter space. We develop bifurcation diagrams showing various patterns of nonspreading solutions from stable equilibria, period two, to chaos. We show that from a relatively uniform initial density with small stochastic perturbations a population consisting of multiple isolated patches can emerge. In ecological terms this work suggests a novel endogenous mechanism for the creation of patch boundaries.
\end{abstract}

Key words. Integro-difference equation, Allee effect, Overcompensation, Nonspreading solution.

AMS subject classification. 92D40, 92D25

Abbreviated title. Nonspreading Solutions in Integro-Difference Equation.

\title{
1 Statements and Declarations
}

- G. Otto did not receive funding to assist in the preparation of this manuscript.

- W.F. Fagan was supported by the National Science Foundation under Grant DMS-1853465.

- B. Li was partially supported by the National Science Foundation under Grant DMS1951482.

- The authors have no relevant financial or non-financial interests to disclose.

\section{Introduction}

As spatial ecology has developed, a great variety of mathematical modeling approaches have been used to study questions at various levels of complexity. Integro-difference equations, which feature a continuous space but discrete time formulation, have proven especially useful for studying questions about population-level processes and species interactions. For example, integro-difference models have been used to predict changes in gene frequency $[33,34,35,45$, 51], and characterize species' spatial dynamics [18, 19, 20, 21, 24, 25, 26, 31, 39]. Because integro-difference equations often admit traveling wave solutions of various kinds, a primary focus in many of these studies has been spatial spread (e.g., expansion of a population or a 
favorable allele). Examples include scenarios in which population fronts can expand spatially in an accelerating fashion [27] and cases where one or more species can (or cannot) outrun the pace of environmental change [22, 30, 53].

Here, we adopt a very different perspective in that we use an integro-difference formulation to study non-spreading solutions. Roughly speaking, a nonspreading solution is a solution which persists in a virtually bounded domain for all generations without expanding its spatial range. Such a solution describes 'invasion pinning' that has been investigated for coupled ordinary differential systems in a discrete (patch) environment (see Keitt et al. [23] and references therein). Similar results can emerge for partial differential equations when the focus is on gap-crossing ability in heterogeneous landscapes, leading to 'geographic range margins' beyond which the species cannot spread [13].

As we discuss below, non-spreading solutions hinge on the existence of so-called Allee effects and overcompensation in the population dynamics of the spatially distributed species. An Allee effect arises when the per-capita birth rate increases as a function of population density when population density is small. Overcompensation is said to occur when high population densities result in a net decrease in offspring production. Allee effects may occur via a great many biological mechanisms $[1,2,4,5,6,9,10,11,12,17,32,37,42,46]$, and they have been studied in connection with integro-difference equations in the context of spatial spread [28, 50]. A special kind of Allee effect, termed a strong Allee effect, occurs when there is a critical population density below which extinction occurs. Mating failure, which can arise through mechanisms like pollen limitation and reproductive asynchrony [6,17], has been linked to strong Allee effects in diverse biological systems. For example, in evergreen bagworms (Thyridopteryx ephemeraeformis), the intensity of a strong Allee effect arising from mating failure is a function of climate, and this spatial variation leads to a hard geographic boundary for the species [36, 43].

We consider the spatial-temporal dynamics of populations governed by the integro-difference equation

$$
u_{n+1}(x)=\int_{-\infty}^{\infty} k(x-y) g\left(u_{n}(y)\right) \mathrm{d} y,
$$

where $u_{n}(x)$ is the density of individuals at point $x$ and time $n, g(u)$ describes density dependent fecundity, and $k(x-y)$ is the dispersal function, which depends upon the distance $|y-x|$ between the location of birth $y$ and the location of settlement $x$. Model (1) describes that individuals at location $y$ generate $g\left(u_{n}(y)\right)$ offspring and then die and these offspring disperse to location $x$ with the probability $k(x-y)$. We will assume that $g(1)=1$, so that the population has an equilibrium at the carrying capacity $u_{n}(x) \equiv 1$, and that $k(x)$ decays at least exponentially fast near $\pm \infty$ so that the probability that an individual travels a very long distance is small.

For the case that small populations grow (i.e., $g^{\prime}(0)>1$ ), the reproduction function $g(u)$ exhibits no Allee effect (i.e., $g(u) \leq g^{\prime}(0) u$ ), and $g(u)$ may or may not produce overcompensation (i.e., 
$g(u)$ decreases for $u$ past a certain population density), the population will spread at a constant asymptotic spreading speed that can be characterized as the slowest speed of a class of traveling waves (Weinberger [52], Li et al. [29]). In this case, the wave is pulled by the leading edge of invasion. When the reproduction function produces overcompensation, oscillations are generated in the population density behind the wave front ( $\mathrm{Li}$ et al. [29]). Constant spreading speed also occurs if $g(u)$ exhibits a strong Allee effect (i.e., $\left.g^{\prime}(0)<1\right)$ and $g(u)$ is increasing (i.e., there is no overcompensation). In this case, the spreading speed is the unique speed of traveling waves connecting zero and the carrying capacity (Lui [35]), and the sign of the wave speed is the same as that of $\int_{0}^{1}[g(u)-u] d u$ (Wang et al [50]). If $\int_{0}^{1}[g(u)-u] d u>0$, the traveling wave moves forward, if $\int_{0}^{1}[g(u)-u] d u<0$, the traveling wave moves backward, and if $\int_{0}^{1}[g(u)-u] d u=0$ the traveling wave is stationary. The wave speed depends on the forward pushing force developed by the high-density populations above the Allee threshold behind wave front as well as the backward pulling force generated by the lower-density populations below the Allee threshold along the leading edge of invasion.

Fluctuating invasion speeds can be generated by a strong Allee effect and strong overcompensation (Sullivan et al. [48]). Strong overcompensation in general produces large spatiotemporal variation in density behind the invasion front and thus, variation in the strength of the push, leading to oscillating spreading speeds. As pointed out in [48], where the population density is smaller than the Allee threshold along the leading edge of the invasion, the population declines before the next time step. Populations above the Allee threshold will grow until they surpass the overcompensation threshold. If the overcompensation is strong enough they will return from a high level to a low level resulting in cyclical variability in the pushing strength of the wave.

In this paper we further study the effects of a combination of a strong Allee effect and strong overcompensation. We will show that such a combination can produce biologically meaningful robust nonspreading solutions in solid regions of the parameter space for (1). We will demonstrate the existence of robust nonspreading solutions with a variety of spatiotemporal patterns. One of our novel findings is the existence of non-spreading solutions that oscillate in both density and spatial range. Here in the long run, the oscillating forward pushing force developed by overcompensation is balanced by the backward pulling force from populations below the Allee threshold, leading to persisting nonspreading solutions. It should be pointed out that, as discussed above, for the case of no overcompensation, there exists a traveling wave with zero speed if $\int_{0}^{1}[g(u)-u] d u=0$; however this condition is not robust and is not satisfied with a slight change of model parameters. It is surprising to note that simple scalar integro-difference equations designed as a modelling tool to investigate spatial spread of populations possess biologically meaningful solutions that actually do not spread in a homogeneous continuous environment. One consequence of the existence of nonspreading solutions concentrated on effectively bounded domains is the formation of multiple population 'patches' separated from each other in space. This will be also explored in the present paper. 


\section{Non-Spatial Model}

We will model growth using the two parameter function presented by Vortcamp et al. [49]. In this growth function $a$ represents the Allee threshold and $r$ represents a parameter controlling the strength of over-compensation. By appropriate scaling of $u$ the carrying capacity can be assumed to be 1 . The growth function used in model (1) is then

$$
g_{a, r}(u):=u \exp \left(r(1-u)\left(\frac{u}{a}-1\right)\right) .
$$

Increasing $r$ for fixed $a$ increases the maximum value of $g_{a, r}(u)$ and accordingly increases the magnitude of the derivative at the equilibria $u=a$ and $u=1$ (Supplementary Materials, Fig. 8). We see that decreasing $a$ for fixed $r$, increases the maximum value of $g_{a, r}(u)$. It can also be seen that in this parametrization the shape of $g_{a, r}(u)$ is more sensitive to the parameter $a$ than $r$.

Essential extinction is said to occur when the image of the maximum value of $g_{a, r}(u)$ is mapped below the Allee threshold [44]. This region in parameter space is indicated in green in Fig. 4(a). It is known that for monotone growth functions with an Allee effect that the sign of population spread is indicated by the sign $\int_{0}^{1}\left[g_{a, r}(u)-u\right] \mathrm{d} u$ [50]. While this is not necessarily true for non-monotone functions, we can still interpret the negativity of the integral as an indication of overall weak growth. The region in parameter space with weak growth is indicated in blue in Fig. 4(a).

\section{Dispersal Kernel}

It is long been known that the shape of the dispersal kernel, particularly its kurtosis, can have a profound influence on the the spreading dynamics in an integro-difference equation [27]. To model dispersal with varying kurtosis we will use the generalized Gaussian distribution centered at the origin with standard deviation 1. Spatial coordinates can trivially be rescaled to satisfy that the standard deviation is 1 without altering the dynamics of the integro-difference equation. The kurtosis of the distribution is controlled by the parameter $\eta>0$. The probability density function used in model (1) for dispersal is then 


$$
\begin{aligned}
& k_{\eta}(x)=C \exp \left(-\left|\frac{x}{S}\right|^{\eta}\right) \\
& \text { where } C=\sqrt{\frac{\Gamma\left(\frac{3+\eta}{\eta}\right)}{12 \Gamma\left(\frac{1+\eta}{\eta}\right)^{3}}} \\
& \text { and } S=\sqrt{\frac{3 \Gamma\left(\frac{1+\eta}{\eta}\right)}{\Gamma\left(\frac{3+\eta}{\eta}\right)}}
\end{aligned}
$$

Where $\Gamma(\cdots)$ refers to the gamma function.

Kurtosis, which is defined as the ratio of the fourth moment to the square of the second, gives a measure of the "fatness" of the tail of the distribution. Leptokurtic distributions $(0<\eta<2)$ can be thought of as having most individuals disperse very small distances with a few individuals dispersing extreme distances in such a way the standard deviation remains fixed. Conversely, platykurtic distributions $(\eta>2)$ can be thought of as most individuals dispersing about the same distance. When $\eta=1,2$, and $\infty$, the commonly used Laplace, Gaussian and Uniform distribution are recovered as is shown in Fig. 9 in the Supplementary Materials.

\section{Spatial Model}

The spatial model is specified by model (1) with the definitions of $g_{a, r}(u)$ and $k_{\eta}(x)$ previously outlined. The inital data is specified by

$$
u_{0}(x)= \begin{cases}p_{0} \cos \left(\frac{\pi}{w_{0}} x\right) & |x|<\frac{w_{0}}{2} \\ 0 & \text { otherwise }\end{cases}
$$

where $p_{0}$ is the maximum density and $w_{0}$ is the width of the support. The solution set $\left\{u_{n}(x)\right\}_{n=0}^{\infty}$ is thus fully specified by the 5 parameters $a, r, \eta, p_{0}, w_{0}$ together with model (1).

To numerically generate the solution set we uniformly discretize space using a step size $\delta=$ 0.005 and use conv in Matlab to compute the accelerated convolution. We use the symmetry of $u_{n}(x)$ about $x=0$ to further accelerate calculations. Both the vector representing $k(x)$ and $u_{n}(x)$ are clipped where they fall below $10^{-5}$. It should be noted that this clipping of $k(x)$ only very minorly effects the computed value 0 th, 2 nd and 4 rth order moments provided $\eta \gtrsim 0.5$. 


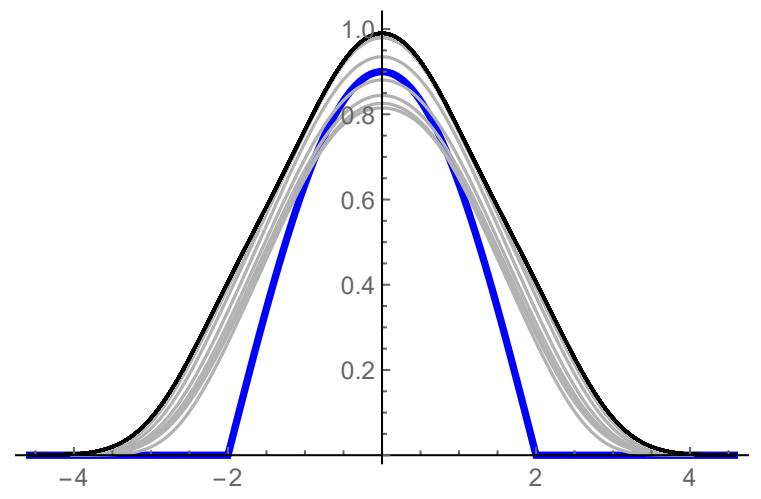

(a) density curves

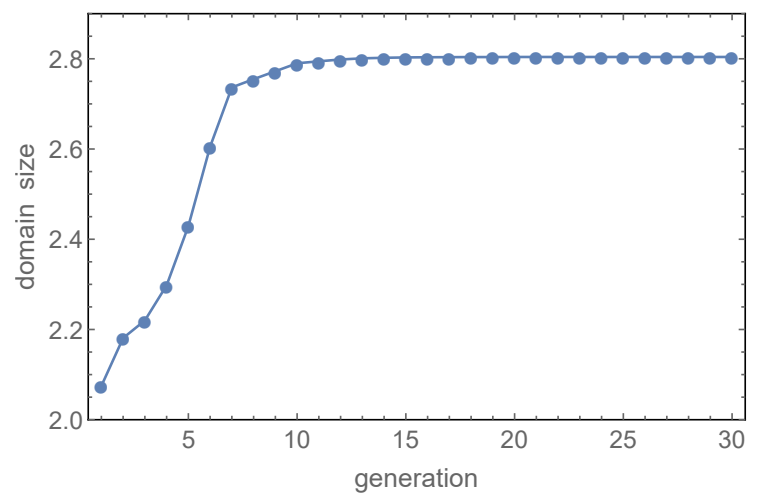

(b) domain size

Figure 1: Solution with parameters $a=0.62, r=8, \eta=5, p_{0}=0.9, w_{0}=4$. In part (a) the blue curve is $u_{0}(x)$, and the gray curves are the transients $u_{1}$ through $u_{15}$ and the black is $u_{16}$ through $u_{100}$. In (b) we see the domain size converging to that of the stable equilibrium.

\section{Non-Spreading Solutions}

In contrast to integro-difference equations with Allee or over-compensation effects considered separately [29, 35, 50], we are able to find solid regions of parameter space with solutions where the population persists but is effectively confined to a limited region of space. As a demonstration, in Fig. 1 we see an example of a solution converging to a stable equilibrium where the population is effectively is limited to $-4 \leq x \leq 4$. More complex behavior such as period 2 and non-periodic non-spreading solutions can be observed as well (Supplementary Material Fig. 10, 11). It should be noted that throughout this paper, we define domain size to be

\subsection{Parameter Bifurcations for Non-spreading Solutions}

Below we present parameter bifurcation diagrams centered on the parameter set $a=0.61, r=$ $8, \eta=5, p_{0}=1, w_{0}=6$. The bifurcations around this set of parameters are typical of those made for other choices. The $x$-axis is the bifurcation parameter, and the $y$-axis is the domain size of the density curve $u_{n}(x)$. Only the domain size of iterations 200-400 are plotted.

In Fig. 2 we see a period doubling bifurcation in the parameter $a$. For values of $a$ between .603 and .64 we see a single period 1 solution emerges; for values between 0.58 and 0.603 a period 2 solution emerges; and for values approximately between 0.575 and 0.58 chaotic dynamics occur. Extinction occurs for small and large values of $a$, and it can be seen regions of extinction are intermingled with non-spreading solutions for $a$ between 0.58 and 0.6.

In Fig. 3 we show the bifurcation for $\eta$, which is the parameter controlling the kurtosis of 
dispersal. We see extinction occurs for leptokurtic dispersal $(\eta<2)$, period 2 solutions occur for $\eta$ slightly higher than 2 and less than 4 , and a period 1 equilibrium for highly platykurtic dispersal when $\eta>4$.

Bifurcation diagrams for $r, p_{0}$ and $w_{0}$ can be viewed in Fig. 12, 13, 14 in the Supplementary Materials.

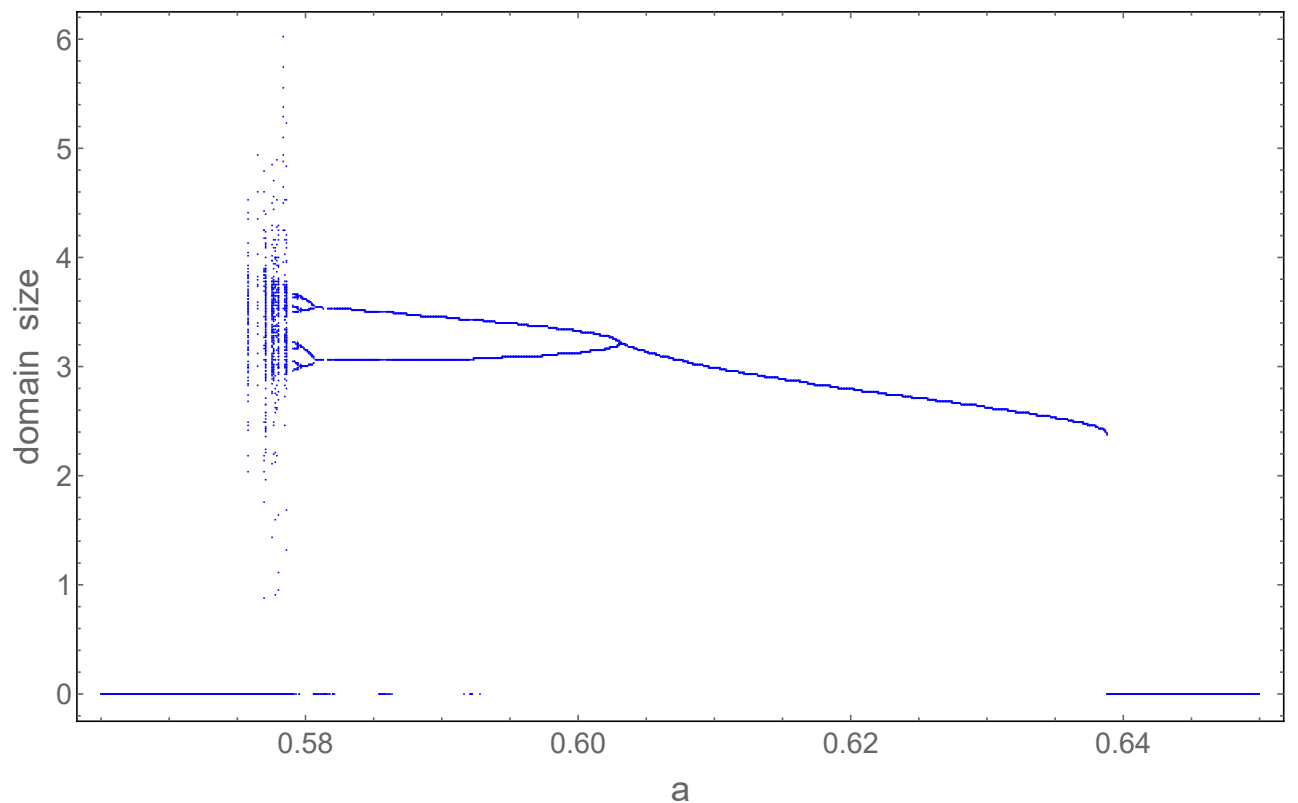

Figure 2: Bifurcation diagram for parameter $a$ with $r=8, \eta=5, p_{0}=1, w_{0}=6$.

\subsection{Classification Map of Parameter Space for Non-spreading Solutions}

The non-spreading solutions exist along a fairly narrow band in parameter space centered around the curve where $\int_{0}^{1}\left[g_{a, r}(u)-u\right] \mathrm{d} u=0$ (see Fig. 4). As was previously mentioned, the maximum value of $g_{a, r}(u)$ is highly sensitive to the parameter $a$ and the narrowness of the region might be considered an artifact of the parametrization.

To numerically classify the parameter space, we divided the region of the $a-r$ plane depicted in Fig. 4(b) into a $100 \times 100$ grid. For the values of $a, r$ on the grid, iterations are computed using $\eta=5, p_{0}=1, w_{0}=6$. The following are used as criteria for classification:

- If the max value of $u_{n}(x)<a$ the solution is classified as extinction (gray).

- If the domain size (see 4.1 for definition) exceeds 24 in less than 500 iterations, the solution is classified as spreading (yellow).

- If periodicity is detected or the domain size fails to exceed 24 in 500 iterations the solution is classified as non-spreading (blue). 


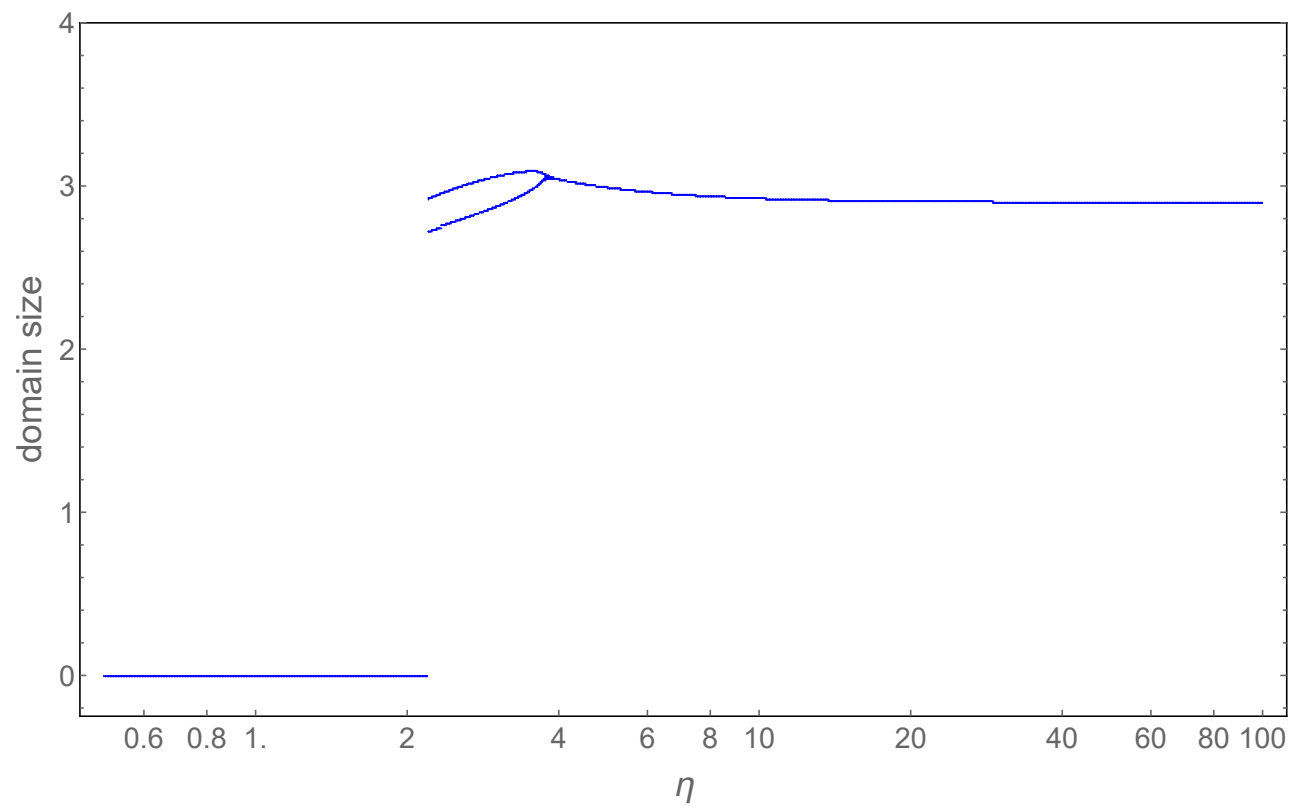

Figure 3: Bifurcation diagram for parameter $\eta$ with $a=0.61, r=8, p_{0}=1, w_{0}=6$. Note that the scale on the $x$-axis is logarithmic.

In Fig. 5 we examine the dependence on initial conditions by varying the parameters $w_{0}$ and $p_{0}$ in model (1). Predictably extinctions occur if the support of the initial domain is too small (small $w_{0}$ ), or if the initial density is too small $\left(\right.$ small $\left.p_{0}\right)$. We see a single non-spreading solution (blue region of Fig. 5) emerges fairly robustly for values of $w_{0}$ not extremely small or large compared to the patch size, and $p_{0}$ above the Allee threshold but not too high above the carrying capacity. For very large values of $w_{0}$ multiple isolated patches, which are essentially copies of a single patch such as is shown in Fig.1, can emerge as the solution. In Fig. 5 we see that for large $w_{0}$ and $p_{0}$, two patches will emerge. This can be attributed to the intermediate population densities which produce the maximum amount of offspring being located at opposing ends of the initial distribution. An equilibrium two-patch density curve is shown in Fig. 6. The resultant equilibrium is qualitatively similar for all red areas in Fig. 5, although the spacing between patches may differ.

\subsection{Patch Formation with a Stochastic Initial Condition}

We wish to simulate a spatially stochastic initial condition with spatial correlation length of $L_{\text {scale }} . L_{\text {scale }}$ can be considered as the length whereby statistical correlations in density diminish to insignificant levels. To generate this initial condition we use that the value of $u_{0}(x)$ is an independent identically distributed random variable uniformly distributed on $[0.8,1.2]$ for $x$-values that are multiples of $L_{\text {scale }}$. For values of $x$ that are not multiples of $L_{\text {scale }}$, values are linearly in- 


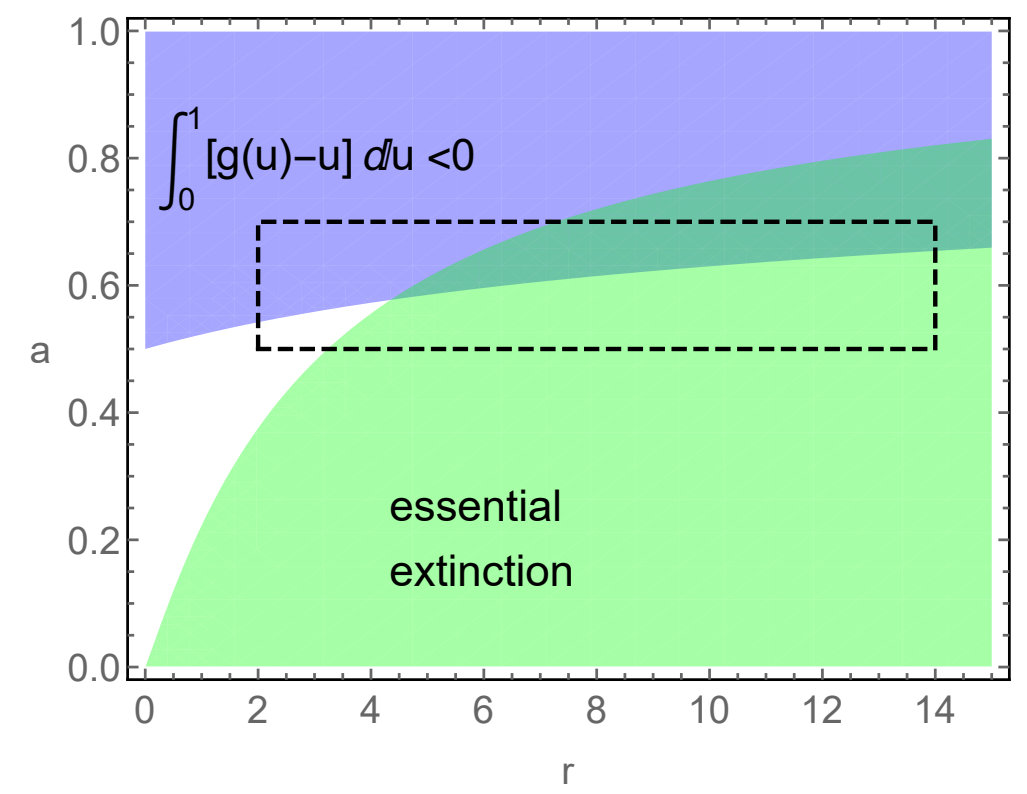

(a) The growth parameter space showing regions of low growth (blue), and essential extinction (green).

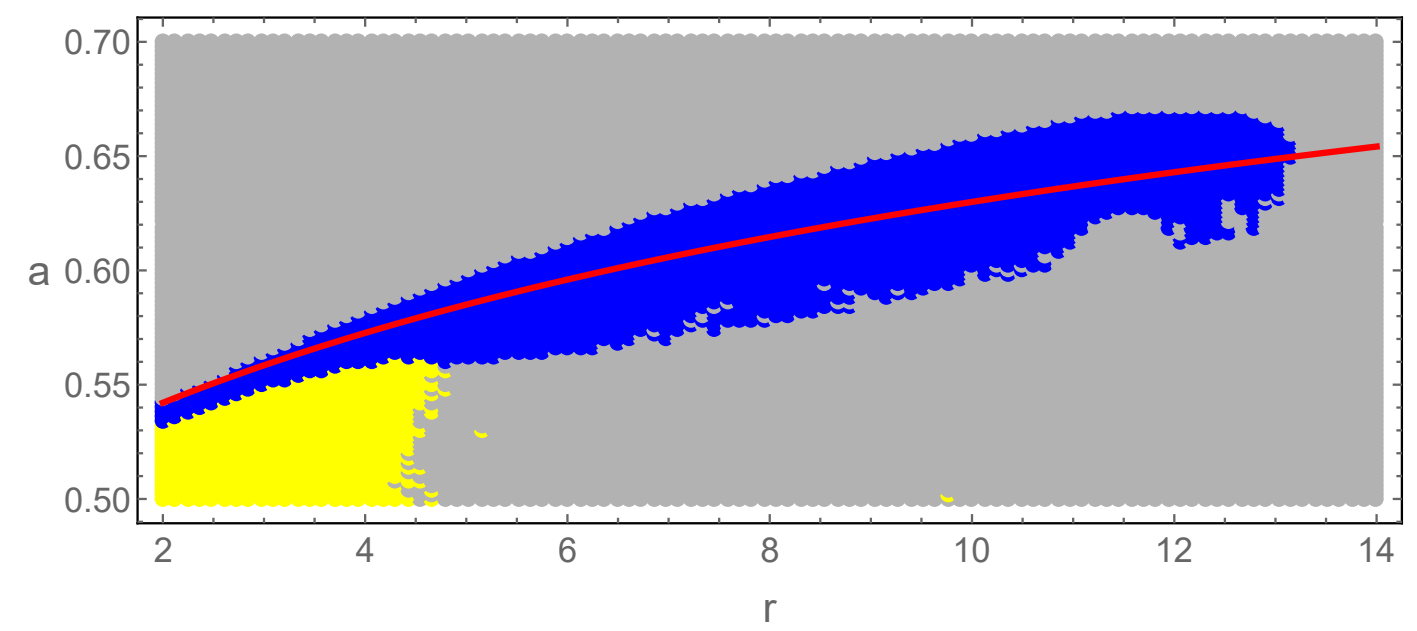

(b) A color map classifying the behavior of model (1) in $a, r$ parameter space. Dark blue regions are non-spreading solutions, and yellow are spreading solutions. The solid red curve is where $\int_{0}^{1}(g(u)-$ $u) \mathrm{d} u=0$.

Figure 4: In sub-figure (a) the region in the black dashed box is shown in detail in sub-figure (b). The other parameter values used in (b) are $\eta=5, p_{0}=1, w_{0}=6$.

terpolated from the control points $\left(n L_{\text {scale }}, u_{0}\left(n L_{\text {scale }}\right)\right)$. We limit our study to parameter values $a=.61, r=8, \eta=5$.

To study the effects of $L_{\text {scale }}$ on patch formation we:

- Used a domain of length 500 initiated as described above. 


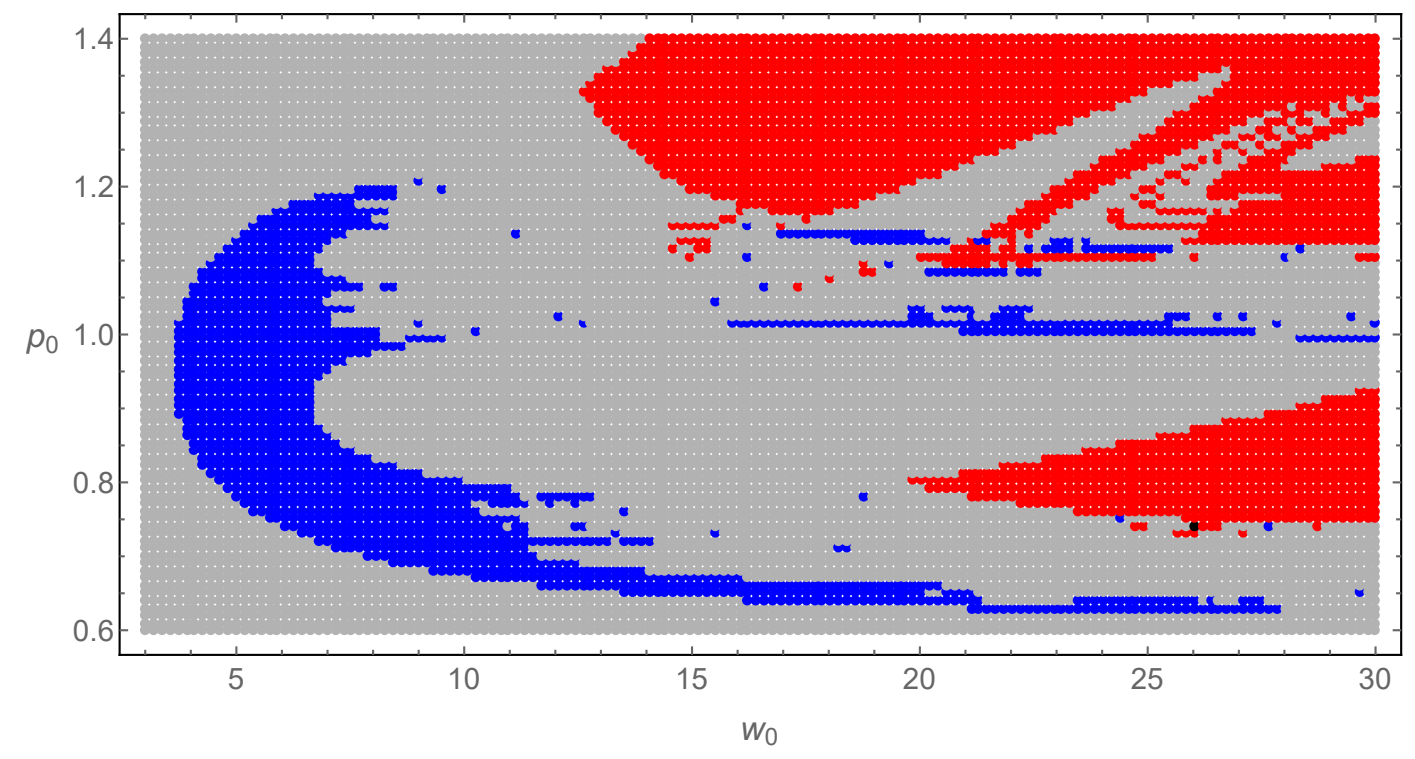

Figure 5: Initial condition parameters resulting in a single patch are shown in blue. Red represents parameters giving rise to two patches. Gray represents parameters resulting in extinction. Values used for other parameters are $a=.61, r=8, \eta=5$.

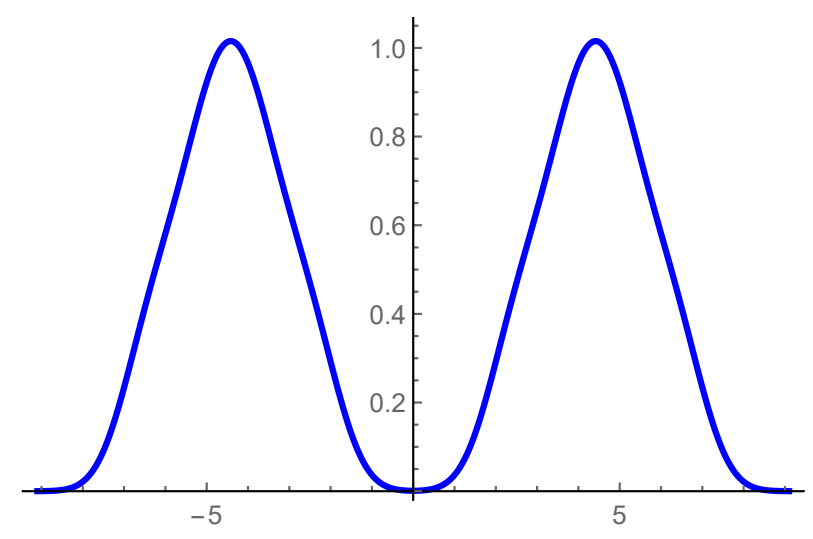

Figure 6: The two-patch equilibrium population density curve for $a=.61, r=8, \eta=5, p_{0}=$ $1.3, w_{0}=17$.

- Iterated until a fixed point condition was met.

- Counted the number of patches formed by integrating the total population and dividing by the population of a single patch.

- For values of $L_{\text {scale }}=0.01,0.05, \cdots, 50,100$, twenty trials were completed for each value.

- $90 \%$ confidence intervals are computed using the assumption of normality (Student T distribution). It should be noted that the sample standard deviation for patch formation was 
about 3 patches relatively independent of $L_{\text {scale }}$.

The length scale of a single patch is $\sim 8$ (see Fig.1). As patches cannot overlap, the maximum possible density of patch formation is $\sim \frac{1}{8}$. In Fig. 15 we give the density of patch formation (\# of patches formed/500) as a ratio to this maximum theoretical value.

We see that for about 3 orders of magnitude, $0.1<L_{\text {scale }}<10$, the density of patch formation saturates at around $25 \%$ of the maximum possible density. The reason it does not come closer to $100 \%$ appears to be that slight overlap of patches usually results in both patches annihilating. For large values of $L_{\mathrm{scale}}$ efficiency of patch formation drops, presumably due to the mild gradients causing the dynamics to be similar to the spatially uniform case where essential-extinction results. Finally, for $L_{\text {scale }}$ much less than the scale of the dispersal distance $\left(\sigma^{2}=1\right)$ we see the efficiency of patch formation again drops. Since dispersal sharply drops the amplitude of the Fourier components with wavenumber much greater than the dispersal length, we see the 1 st generation will be an almost constant density whose value will be the average of $g(u)$ over $0.8<u<1.2$. Thus again the non-spatial dynamics will be dominant. 


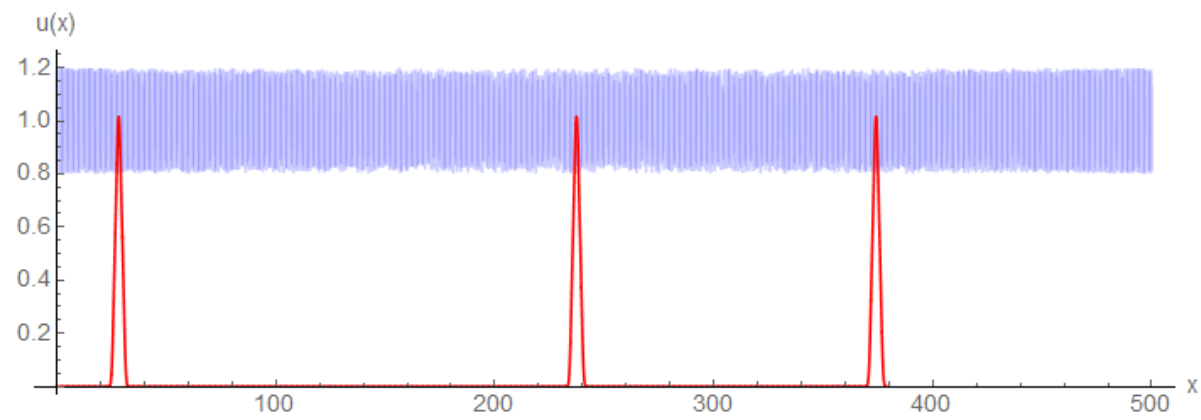

(a) $L_{\text {scale }}=0.01$

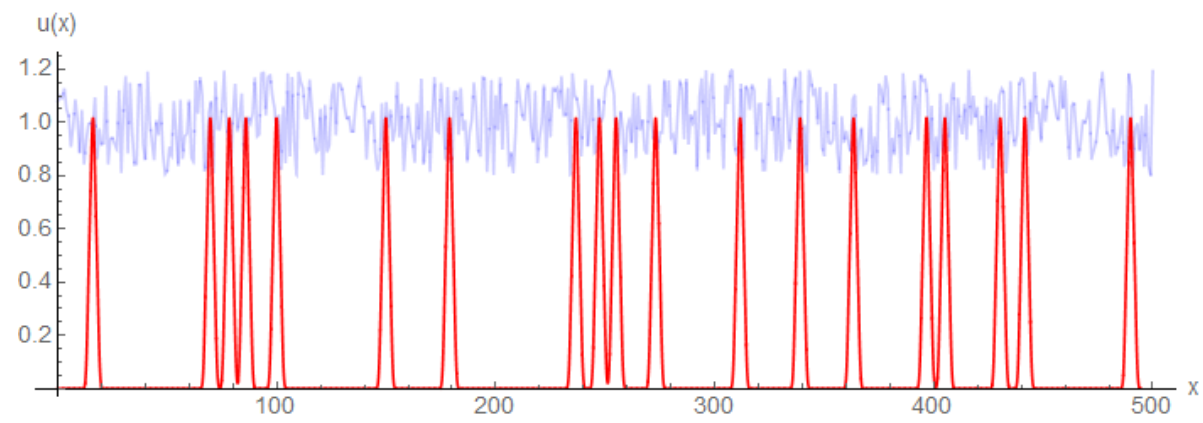

(b) $L_{\text {scale }}=1$

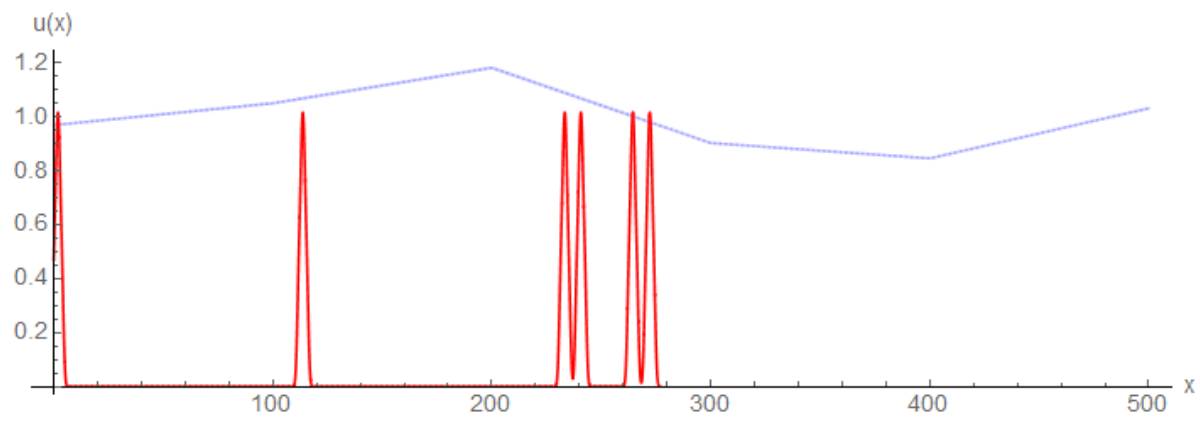

(c) $L_{\text {scale }}=100$

Figure 7: Typical plots of initial conditions (blue) and resulting patch formation (red) at short, intermediate, and long length scales.

\section{Discussion}

In this paper we studied non-spreading solutions for the integro-difference equation (1) where the growth function $g(u)$ exhibits a strong Allee effect and overcompensation. The non-spreading solutions take forms of stable equilibrium solutions vanishing at $\pm \infty$ and solutions oscillating in densities and spatial ranges. Such nonspreading solutions exist in a solid region in the parameter space. In a large habitat, patch formation can be developed with each patch essentially formed by a non-spreading solution. Our results show that single species model (1) with constant parameters can have very rich nonspreading population dynamics. 
Both a strong Allee effect and overcompensation in population growth are necessary to produce biologically meaningful nonspreading solutions. Overcompensation generates a forward pushing force from populations above the Allee threshold. Likewise, a backward pulling force is created from populations below the Allee threshold. Nonspreading solutions emerge when there is a balance between the forward pushing and backwards pulling forces. The existence of oscillating non-spreading solutions is due to a forward pushing force derived by variation in density behind the population distribution front. In the absence of overcompensation, model (1) with a strong Allee effect can have a non-spreading solution if and only if $\int_{0}^{1}[g(u)-u] d u=0$, so that such a solution exists only in a region with measure zero in the parameter space, and thus it is not robust. However, as indicated in the bifurcation diagram Figure 13, with both a strong Allee effect and overcompensation, there is a solid parameter region (blue) in which non-spreading solutions exist. In this region, $\int_{0}^{1}[g(u)-u] d u$ can be greater than 0 , less than 0 , or 0 . The bifurcation diagram Fig. 2 shows various patterns of nonspreading solutions from steady states, period two, to chaos when $a$ varies while other are fixed. It should be noted that $\eta$, the kurtosis of the dispersal kernel, and initial data also play important roles in developing nonspreading solutions. In Fig. 3, there is no nontrivial nonspreading solutions for $\eta<2$, period 2 nontrivial solutions exits on a relatively small interval near $\eta=2$, and for relatively large $\eta$ there is a stable nonspreading equilibrium. In Fig. 13 and 14, nonspreading solutions depend on the amplitudes and support of initial data. In addition to the growth function used within this paper, the PhD Thesis of Otto [41] demonstrates that nonspreading solutions can form with a variety of other forms of growth function.

Nonspreading solutions provide a basis for the development of patch formation. For a large habitat, separate patches can emerge from perturbations in a relatively constant population, with each patch basically a nonspreading solution as is demonstrated in Fig. 7. Patch formation is weakly sensitive to the length scale of correlations in the initial distribution as shown in the Supplementary Materials Fig. 15, with patch formation being favored by length scales on the order of the dispersal distance. Correlation lengths much larger or smaller than the dispersal distance result in inefficient patch formation.

We also found that growth parameters giving rise to essential extinction in the non-spatial model, can actually experience population spread and population growth in the spatial model. This is consistent with Vortkamp et al.[49], who using a spatially discrete 2-patch model demonstrated that essential extinction could be stabilized due to out of phase rescue effect. We will save further investigation of this phenomena for future work.

Our result on non-spreading solutions contrasts with that of Sullivan et al. [48]. Working on (1) with a truncated Ricker's function for population growth, they found that fluctuating spreading speeds can occur as a result of a combination of a strong Allee effect and overcompensation. The scaled growth function $g(u)$ given by (1) with the carrying capacity 1 has two parameters describing the Allee threshold and strength of overcompensation, respectively. If the carrying 
capacity of the truncated Ricker's function is scaled to 1, there is no parameter controlling the strength of overcompensation. Therefore the growth function used in this paper is more flexible than the truncated Ricker's function considered in [48]. Observe that in Figure 4, there also exists a solid region (green) where spreading solutions exist. Depending on the parameters, model (1) possesses spreading and nonspreading dynamics as well as extinction.

The fact that a single mathematical equation can admit such qualitatively divergent output as spreading solutions, non-spreading solutions, and extinction is intriguing. The possibility of non-spreading solutions is particularly interesting because it suggests a new way to connect the widely employed modeling framework of integro-difference equations to a completely different purpose: the origin and maintenance of ecological boundaries. The factors influencing the location and maintenance of species' spatial distributions, whether patch boundaries on small scales or geographic range boundaries on larger scales, have been the subject of intense interest by ecologists for decades $[3,16,47]$. The specific biological mechanisms leading to the existence of such boundaries are diverse, but often reflect an interplay between local population dynamics and dispersal. Such dynamics could be related to the oscillating wave fronts observed with this model (Supplementary Materials, Fig. 10, 11). For example, repeated processes of invasions and extinctions appear to be important for the maintenance of species' patch boundaries in mixed conifer-hardwood forests [15]. Likewise, Allee effects can contribute to the existence of geographic range boundaries in some insect systems with short dispersal distances [36, 43].

Identifying the existence of non-spreading solutions in integro-difference equations opens up several additional lines of inquiry for this modeling framework. One such possibility would involve investigations of how large contiguous populations collapse into small patches, either on evolutionary timescales [38] or in connection with the persistence of relictual populations in conservation biology [7,8]. Likewise, future research could examine non-spreading solutions for integro-difference equations operating on a landscape gradient (e.g., temperature, rainfall) that influences population growth rate. Such studies would provide a vehicle for investigating the interplay between biological and environmental processes that can jointly influence the origin and maintenance of geographic range boundaries [16], including the possibility of patchy population structure at geographic range margins $[3,14]$. Overall, the existence of nonspreading solutions in integro-difference equations suggests the emergence of a welcome new tool for studying diverse phenomena in spatial ecology.

\section{Supplementary Materials}




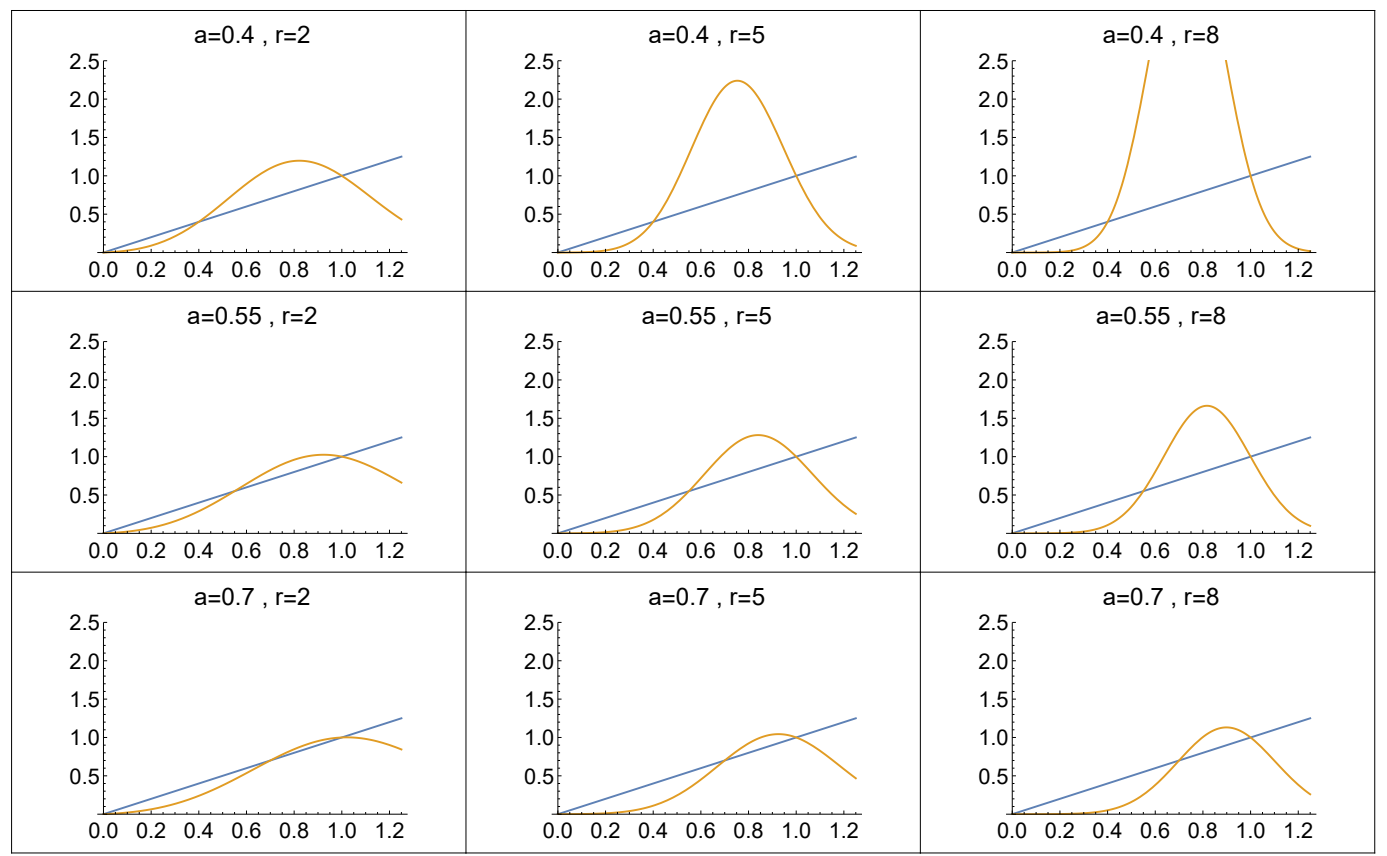

Figure 8: The graph of $g_{a, r}(u)$ and $y=u$ line for various values of $a$ and $r$.

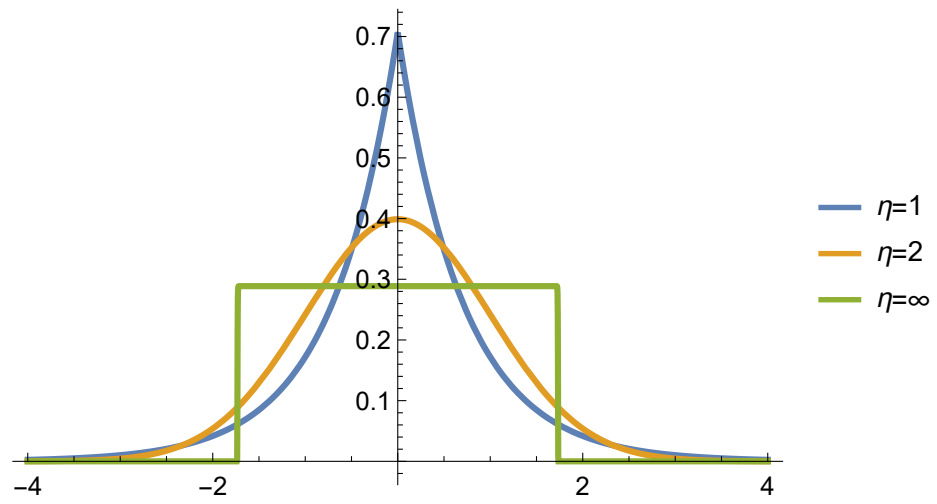

Figure 9: Generalized Gaussian distributions with standard deviation=1 for various values of $\eta$. 


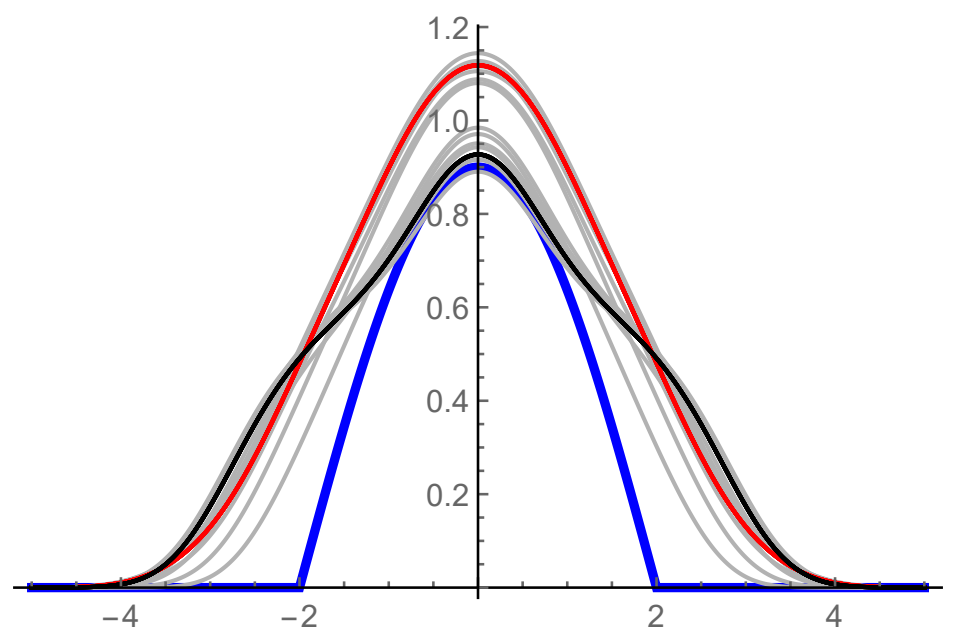

(a) density curves

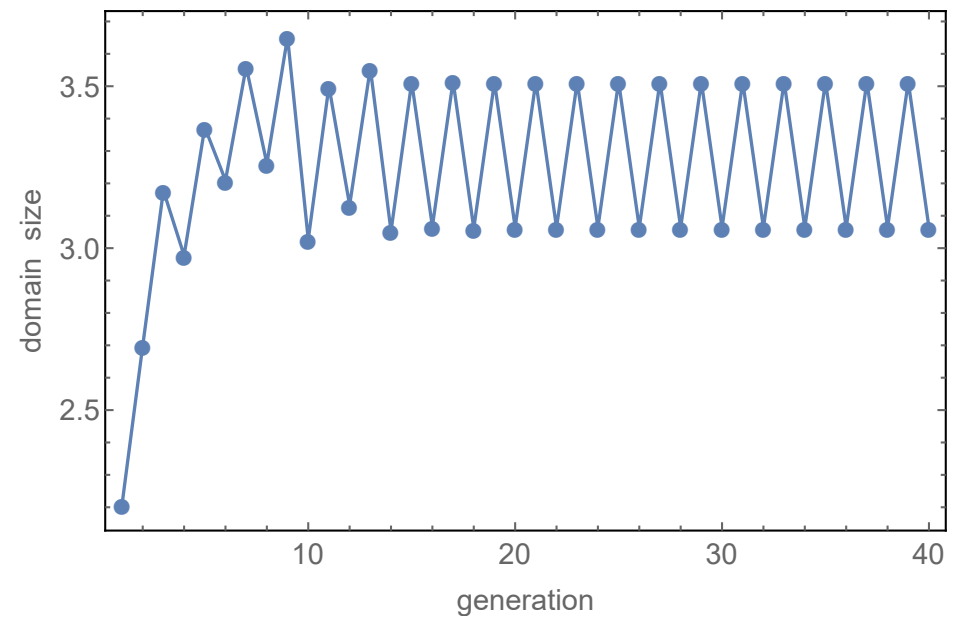

(b) domain size

Figure 10: Solution with parameters $a=0.585, r=8, \eta=5, p_{0}=0.9, w_{0}=4$. In part (a) the blue curve is $u_{0}(x)$, the gray curves are the transients $u_{1}$ through $u_{16}$, the red are the odd indexed iterations $u_{17}$ through $u_{99}$, and the black are the even indexed iterations $u_{18}$ through $u_{100}$. In (b) we see the domain size oscillating with period 2. 


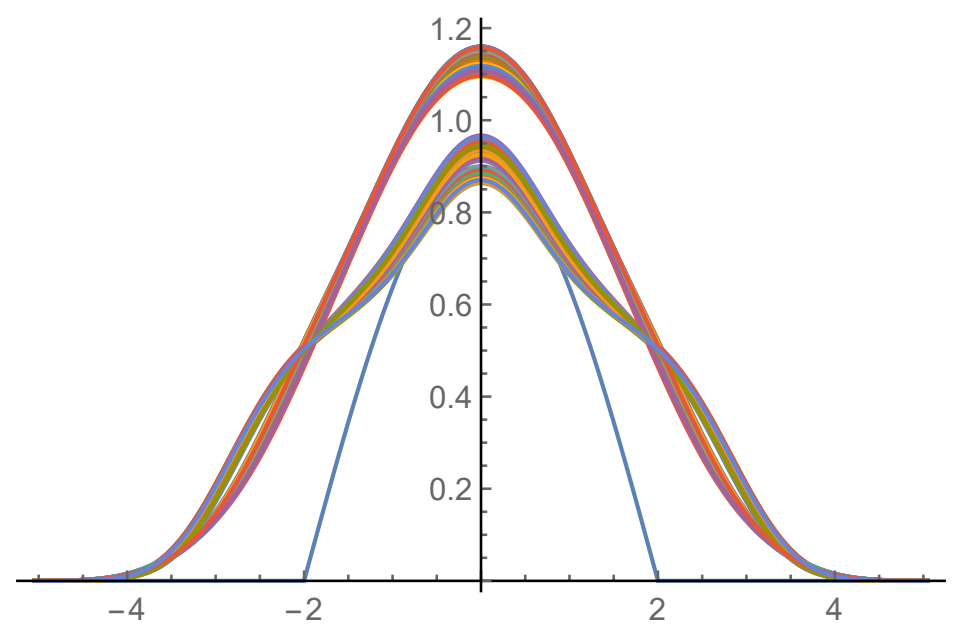

(a) density curves

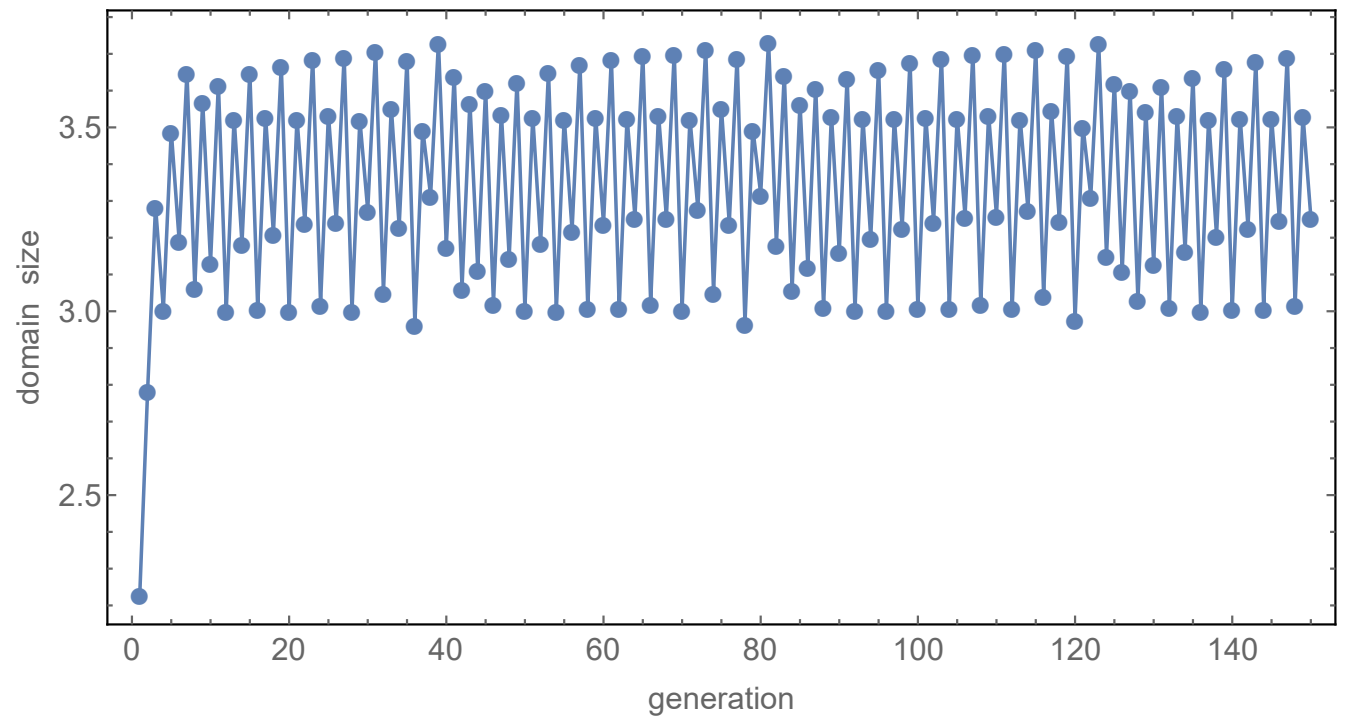

(b) domain size

Figure 11: Solution with parameters $a=0.57865, r=8, \eta=5, p_{0}=0.9, w_{0}=4$. In (a) the blue curve is $u_{0}(x)$, the multicolored curves are $u_{100}$ through $u_{200}$. In (b) we see aperiodic oscillations in the domain size. 


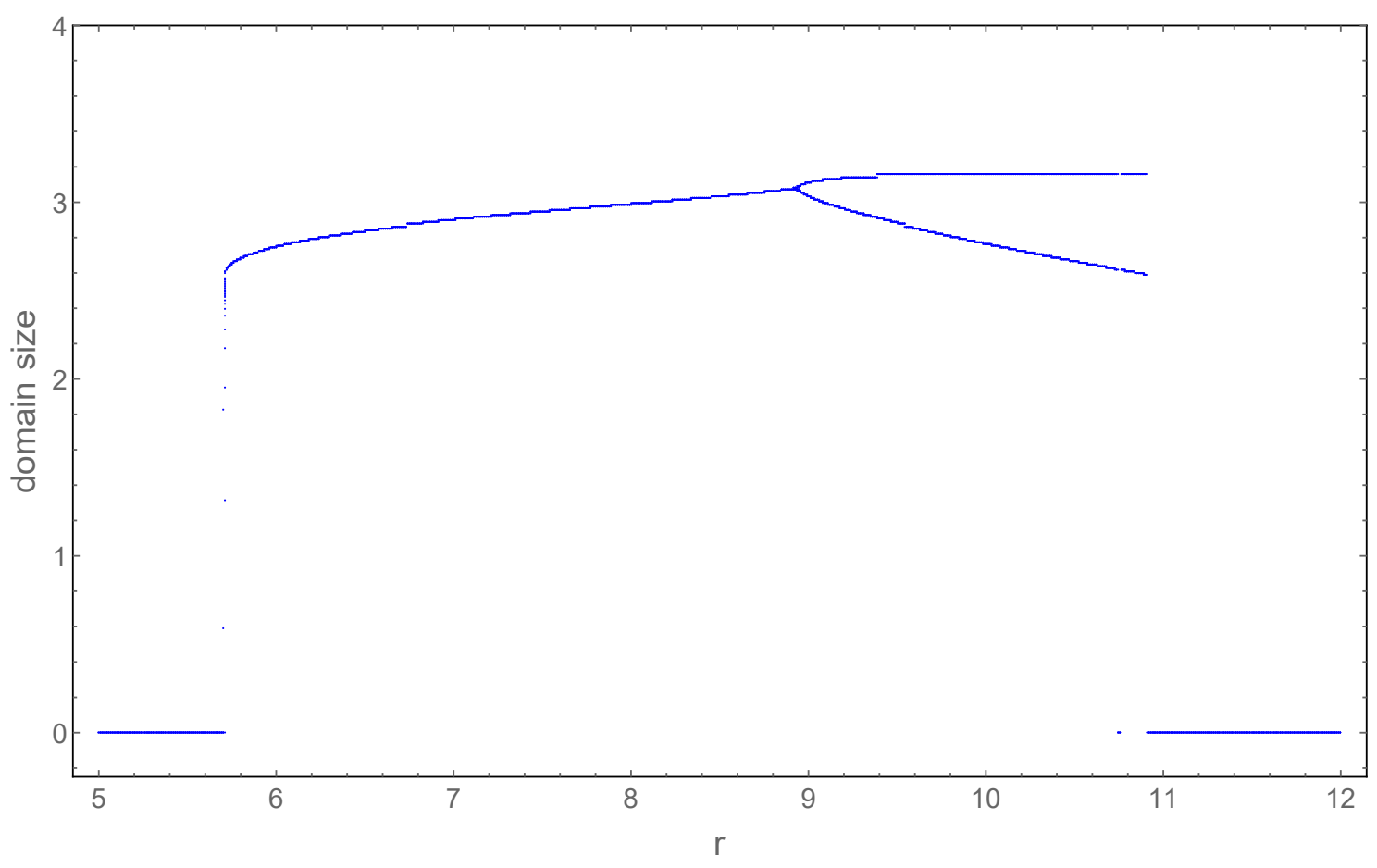

Figure 12: Bifurcation diagram for parameter $r$ with $a=0.61, \eta=5, p_{0}=1, w_{0}=6$.

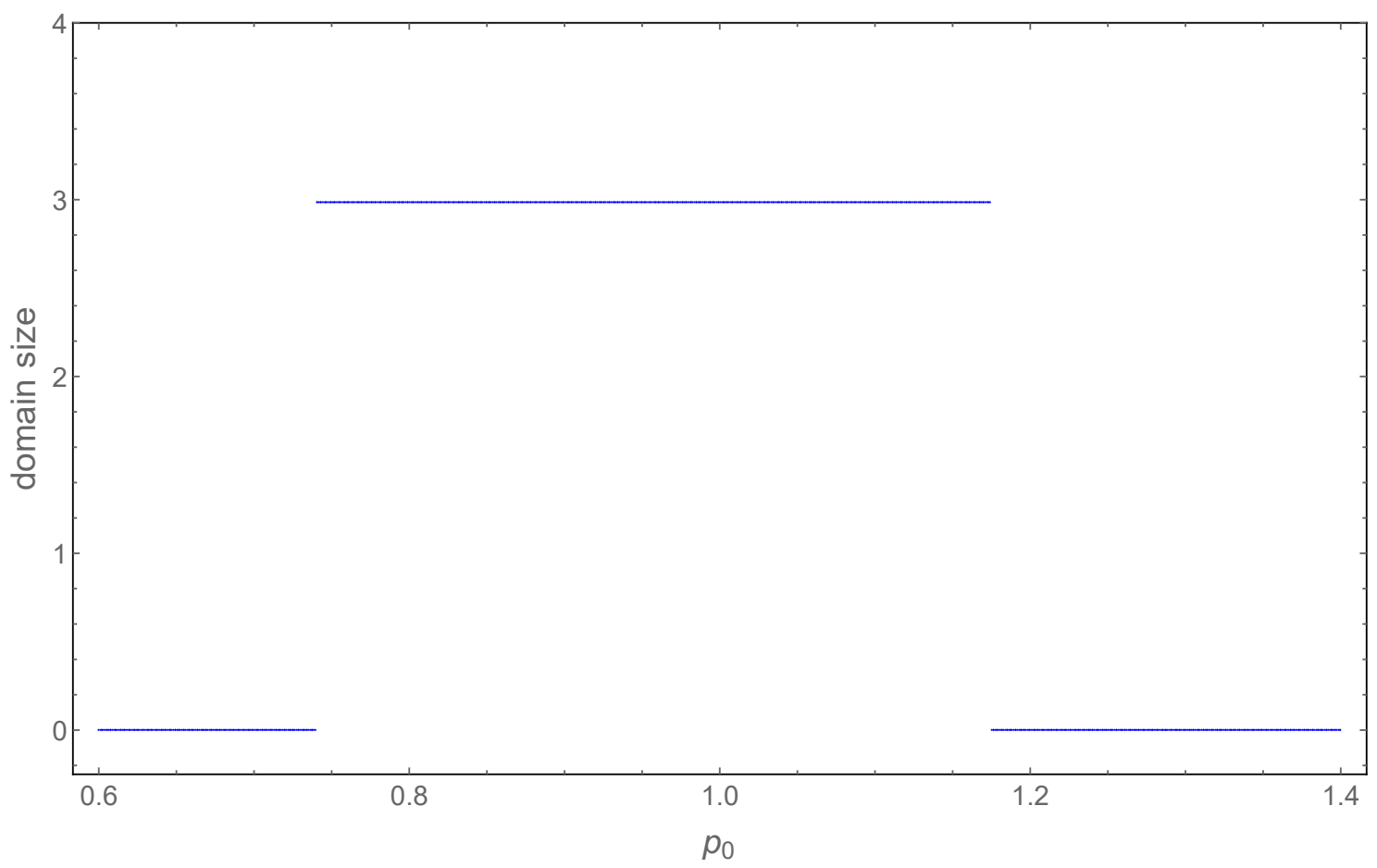

Figure 13: Bifurcation diagram for parameter $p_{0}$ with $a=0.61, r=8, \eta=5, w_{0}=6$. 


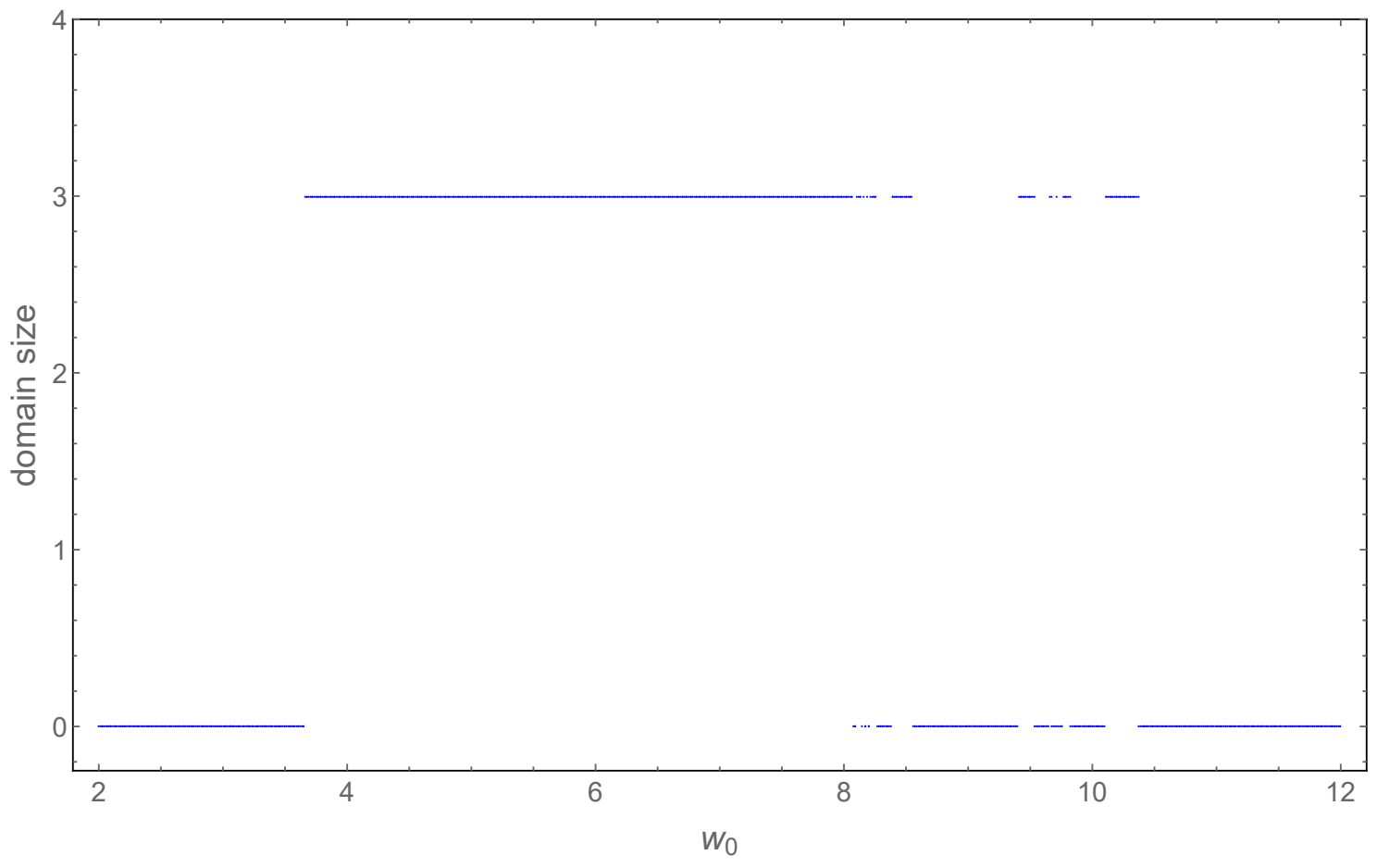

Figure 14: Bifurcation diagram for parameter $w_{0}$ with $a=0.61, r=8, \eta=5, p_{0}=1$.

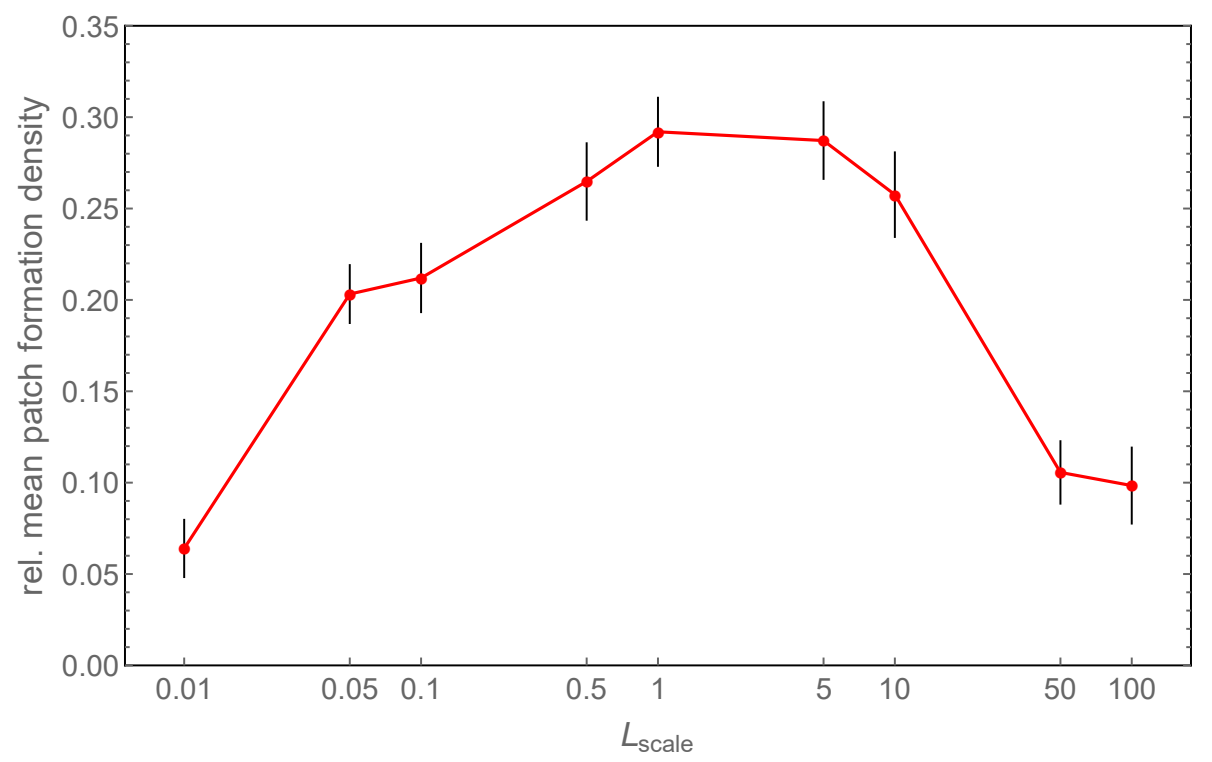

Figure 15: The mean density of patch formation relative to the maximum possible patch density as a function of the correlation length scale of the stochastic initial condition. The black bars are the $90 \%$ confidence intervals. Note the $x$-scale is logarithmic. 


\section{References}

[1] W. C. Allee, A. E. Emerson, O. Park, T. Park, and K. P. Schmidt. 1949. Principles of Animal Ecology, W. B. Saunders, Philadelphia, Pennsylvania, USA.

[2] T.-L. Ashman, T. M. Knight, J. A. Steets, P. Amarasekare, M. Burd, D. R. Campbell, M. R. Dudash, M. O. Johnston, S. J. Mazer, R. J. Mitchell, M. T. Morgan, and W. G. Wilson. 2004. Pollen limitation of plant reproduction: ecological and evolutionary causes and consequences. Ecology 85: 2408-2421.

[3] Brown, J.H., Stevens, G.C. and Kaufman, D.M., 1996. The geographic range: size, shape, boundaries, and internal structure. Annual review of ecology and systematics, 27: 597623.

[4] M. Burd. 1994. Bateman's principle and plant reproduction-the role of pollen limitation in fruit and seed set. Bot. Rev. 60: 83-139.

[5] S. L. Cadre, T. Tully, S. J. Mazer, J.-B. Ferdy, J. Moret, and N. Machon. 2008. Allee effects within small populations of Aconitum napellus ssp. lusitanicum, a protected subspecies in northern France. New Phytologist 179: 1171-1182.

[6] J. M. Calabrese and W. F. Fagan. 2004. Lost in time, lonely, and single: reproductive asynchrony and the Allee effect. American Naturalist 164: 25-37.

[7] Channell, R. and Lomolino, M.V., 2000. Dynamic biogeography and conservation of endangered species. Nature 403: 84-86.

[8] Channell, R. and Lomolino, M.V., 2000. Trajectories to extinction: spatial dynamics of the contraction of geographical ranges. Journal of Biogeography 27: 169-179.

[9] F. Courchamp, L. Berec, and J. Gascoigne. 2008. Allee Effects in Ecology and Conservation. Oxford University Press, London.

[10] F. Courchamp, T. Clutton-Brock, and B. Grenfell. 1999. Inverse density dependence and the Allee effect, Trends in Ecology and Evolution 14: 405-410.

[11] H. G. Davis, C. M. Taylor, J. G. Lambrinos, and D. R. Strong. 2004. Pollen limitation causes an Allee effect in a wind-pollinated invasive grass (Spartina alterniflora). Proc. Natl. Acad. Sci. USA. 101: 13804-13807.

[12] B. Dennis. 1989. Allee effects: population growth, critical density, and the chance of extinction. Natural Resource Modeling 3: 481-537. 
[13] Fagan, W.F., S. Cantrell, C. Cosner, and S. Ramakrishnan. 2009. Interspecific variation in critical patch size and gap crossing ability as determinants of geographic range size distributions. American Naturalist 173: 363-375.

[14] Fortin, M.J., Keitt, T.H., Maurer, B.A., Taper, M.L., Kaufman, D.M. and Blackburn, T.M., 2005. Species' geographic ranges and distributional limits: pattern analysis and statistical issues. Oikos 108: 7-17.

[15] Frelich, L.E., Calcote, R.R., Davis, M.B. and Pastor, J., 1993. Patch formation and maintenance in an old-growth hemlock-hardwood forest. Ecology 74: 513-527.

[16] Gaston, K.J., 2009. Geographic range limits: achieving synthesis. Proceedings of the Royal Society B: Biological Sciences 276: 1395-1406.

[17] M. J. Groom. 1998. Allee effects limit population viability of an annual plant. The American Naturalist 151: 487-496.

[18] D. P. Hardin, P. Takáč, and G. F. Webb. 1988. Asymptotic properties of a continuous-space discrete-time population model in a random environment. Bull. Math. Biol. 26: 361-374.

[19] D. P. Hardin, P. Takáč, and G. F. Webb. 1988. A comparison of dispersal strategies for survival of spatially heterogeneous populations. SIAM J. Appl. Math. 48: 1396-1423.

[20] D. P. Hardin, P. Takáč, and G. F. Webb. 1990. Dispersion population models discrete in time and continuous in space. J. Math. Biol. 28: 1-20.

[21] A. Hastings and K. Higgins. 1994. Persistence of transients in spatially structured ecological models. Science 263: 1133-1136.

[22] C. Hu, J. Shang, and B. Li. 2020. Spreading speeds for reaction-diffusion equations with a shifting habitat, Journal of Dynamics and Differential Equations 32: 1941-1964.

[23] T. H. Keitt, M. A. Lewis, and R. D. Holt. 2001. Allee effects, invasion pinning, and species' borders. Am Nat. 157: 203-216.

[24] M. Kot and W. M. Schaffer. 1986. Discrete-time growth-dispersal models. Math. Biosci. 80: $109-136$.

[25] M. Kot. 1989. Diffusion-driven period doubling bifurcations. Biosystems 22: 279-287.

[26] M. Kot. 1992. Discrete-time traveling waves: Ecological examples. J. Math. Biol. 30: 413436.

[27] M. Kot, M. A. Lewis, and P. van den Driessche. 1996. Dispersal data and the spread of invading organisms. Ecology 77: 2027-2042. 
[28] Lewis M.A., Kareiva P., 1993, Allee Dynamics and the Spread of Invading Organisms, Theo. Pop. Bio. 43: 141-158.

[29] B. Li, M. A. Lewis, and H. F. Weinberger. 2009. Existence of traveling waves for integral recursions with nonmonotone growth functions. J. Math. Biol. 58: 323-338.

[30] B. Li and J. Wu. 2020. Traveling waves in integro-difference equations with a shifting habitat. Journal of Differential Equations 268: 4059-4078.

[31] M. Kot. 2001. Elements of Mathematical Ecology. Cambridge University Press. Cambridge, United Kingdom.

[32] B. M. H. Larson and S. C. H. Barrett. 2000. A comparative analysis of pollen limitation in flowering plants. Biol. J. Linn. Soc. 69: 503-520.

[33] R. Lui. 1982. A nonlinear integral operator arising from a model in population genetics. I. Monotone initial data. SIAM. J. Math. Anal. 13: 913-937.

[34] R. Lui. 1982. A nonlinear integral operator arising from a model in population genetics. II. Initial data with compact support. SIAM. J. Math. Anal. 13: 938-953.

[35] R. Lui. 1983. Existence and stability of traveling wave solutions of a nonlinear integral operator. J. Math. Biol. 16:199-220.

[36] Lynch, H.J., Rhainds, M., Calabrese, J.M., Cantrell, S., Cosner, C. and Fagan, W.F. (2014), How climate extremes - not means_-define a species' geographic range boundary via a demographic tipping point. Ecological Monographs 84: 131-149. https://doi.org/10.1890/122235.1

[37] D. A. Moeller. 2004. Facilitative interactions among plants via shared pollinators. Ecology 85: 3289-3301.

[38] Nekola, J.C., 1999. Paleorefugia and neorefugia: the influence of colonization history on community pattern and process. Ecology 80: 2459-2473.

[39] M. Neubert, M. Kot, and M. A. Lewis. 1995. Dispersal and pattern formation in a discretetime predator-prey model. Theor. Pop. Biol. 48 : 7-43.

[40] M. G. Neubert, M. Kot, and M. A. Lewis. 2000. Invasion speeds in fluctuating environments. Proc. R. Soc. Lond. B. Biol. Sci. 267: 1603-1610.

[41] G. Otto. 2017. Non-spreading Solutiona in a Integro-Difference Model Incorporating Allee and Overcompensation Effects. Ph. D thesis, University of Louisville. 
[42] I. M. Parker. 2004. Mating patterns and rates of biological invasion Proc. Natl. Acad. Sci. USA. 101: 13695-13696.

[43] Rhainds M, Fagan WF. Broad-scale latitudinal variation in female reproductive success contributes to the maintenance of a geographic range boundary in bagworms (Lepidoptera: Psychidae). PLoS One. 2010 Nov 30;5(11):e14166. doi: 10.1371/journal.pone.0014166. PMID: 21152445; PMCID: PMC2994749.

[44] S. J. Schrieber. 2003. Allee effects, extinctions, and chaotic transients in simple population models. Theo. Pop. Bio. 64: 201-209.

[45] M. Slatkin. 1973. Gene flow and selection in a cline. Genetice 75: 733-756.

[46] P. A. Stephens and W. J. Sutherland. 1999. Consequences of the Allee effect for behavior, ecology and conservation. Trends in Ecology and Evolution 14: 401-405.

[47] Strayer, D.L., Power, M.E., Fagan, W.F., Pickett, S.T. and Belnap, J., 2003. A classification of ecological boundaries. BioScience 53:723-729.

[48] L. L. Sullivan, B. Li, T. E. X. Miller, M. G. Neubert, and A. K. Shaw. 2017. Density dependence in demography and dispersal generates fluctuating invasion speeds. Proc. Natl. Acad. Sci. USA. 114: 5053-5058.

[49] I. Vortkamp, S. Schrieber, A. Hastings, and F. Hilker. 2020, Multiple Attractors and Long Transients in Spatially Structured Populations with an Allee Effect. Bull. Math. Biol. 82:82 https://doi.org/10.1007/s11538-020-00750-x

[50] M. H. Wang, M. Kot, and M. G. Neubert. 2002. Integrodifference equations, Allee effects, and invasions. J. Math. Biol. 44: 150-168.

[51] H. F. Weinberger. 1978. Asymptotic behavior of a model in population genetics, in Nonlinear Partial Differential Equations and Applications, ed. J. M. Chadam. Lecture Notes in Mathematics 648: 47-96. Springer-Verlag, Berlin.

[52] H. F. Weinberger. 1982. Long-time beahvior of a class of biological models. SIAM. J. Math. Anal. 13: 353-396.

[53] Zhou, Y., Kot, M. 2011. Discrete-time growth-dispersal models with shifting species ranges. Theor. Ecol. 4: 13-25. 